\title{
Molecular Optomechanical Approach to Surface-Enhanced Raman Scattering in Plasmonic Cavities
}

\author{
$\underline{\text { Javier Aizpurua }}$ \\ Center for Materials Physics in San Sebastián (CSIC-UPV/EHU) and DIPC \\ Paseo Manuel Lardizabal 5, Donostia-San Sebastián 20018, Spain
}

The excitation of molecular vibrations can be efficiently enhanced by the action of optical resonators which improve the interaction between light and matter. Among the variety of optical resonators, plasmonic cavities emerge as a special type which reduce the electromagnetic effective mode volumes down to the nanoscale, thus increasing the coupling strength between molecular vibrations and confined photons. This enhanced interaction brings Surface-Enhanced Raman Scattering (SERS) to a regime where optomechanical effects can be revealed in the dynamics of the inelastic process [1,2]. Furthermore, atomic-scale morphological features in plasmonic cavities are capable to produce extreme light confinement [3], setting sub-nanometric access and control of single-molecular excitations in SERS [4]. To describe the interaction of light and matter in this extreme regime, quantum theoretical frameworks are needed.

The analogy between SERS in molecular spectroscopy and typical optomechanical processes in cavity optics will be described. By adopting an optomechanical hamiltonian which describes the interaction between cavity plasmons and molecular vibrations, it is possible to trace the quantum dynamics of both plasmons and vibrations.

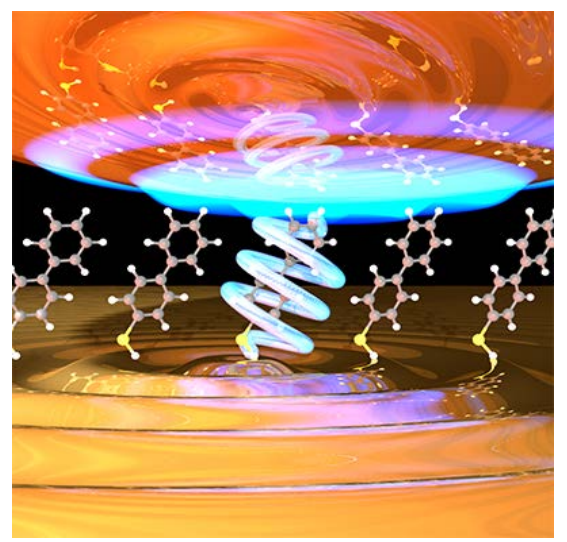

Figure 1 Artistic schematics of a nanometric optical cavity formed by a plasmonic nanoparticle-on-amirror (NPoM) configuration which creates a nanogap with a set of self-assembled biphenyl-4-thiol molecules in it. The induced inhomogeneous plasmonic field at the nanogap is responsible for the emergence of non-linear and coherent effects in molecular fluorescence as well as in vibrational spectroscopy of molecules.

The solution of the master equation of the optomechanical hamiltonian allows for identifying novel nonlinear and coherent effects in SERS [1,2] such as the existence of different regimes of molecular vibrational build-up: (i) a thermal vibrational regime, 
(ii) a vibrational pumping regime, and (iii) a strongly nonlinear vibrational regime, which emerge as a consequence of the dynamics induced by the optomechanical interaction between plasmons and vibrations. Correlations between the Stokes and anti-Stokes Raman signals can also be traced within this model [1].

The presence of these strong optomechanical effects in Raman have been recently addressed experimentally by monitoring the Raman signal from special "picocavities" formed by a few metallic atoms in a plasmonic cavity at cryogenic temperature. The strong optomechanical coupling achieved in this situation is found to activate the pumping regime in the Raman anti-Stokes signal [5]. Furthermore, with the use of pulsed illumination, these effects have also been theoretically described and experimentally monitored in the Stokes signal [6], enabling to bring molecular optomechanics from nonlinear instabilities to bond breaking.

The realm of molecular SERS brings optomechanics to a new regime of energies and dimensions that can be exploited in controlled chemical reactivity and in molecular frequency conversion.

\section{ACKNOWLEDGMENTS}

I would like to acknowledge the members of my group in Donostia-San Sebastián who actively contributed to the contents of this Keynote Talk: Mikolaj Schmidt, Tomas Neuman, and Rubén Esteban, through the Project FIS2016-80174-P of Spanish MINEICO. The experimental results and collaboration with the group of Prof. Jeremy J. Baumberg at the University of Cambridge (U.K.) are also greatly acknowledged.

\section{REFERENCES}

[1] Schmidt MK, et al., ACS Nano 2016, 10, 6291-6298.

[2] Schmidt MK, et al., Faraday Discussions 2017, 205, 31-65.

[3] Urbieta M, et al., ACS Nano 2018, 12, 585-595.

[4] Zhang R, et al., Nature 2013, 498: 82-86.

[5] Benz F, et al., Science 2016, 354, 726-729.

[6] Lombardi A, et al., Phys. Rev. X, 2018, 8, 011016. 